\title{
A Resource Reuse Scheme of D2D Communication Underlaying LTE Network with Intercell Interference*
}

\author{
Jia Liu ${ }^{1}$, Bingbing Li ${ }^{1}$, Bing Lan ${ }^{1}$, Junren Chang ${ }^{2}$ \\ ${ }^{1}$ National key lab of ISN. Xi'an, China \\ ${ }^{2}$ Huawei Technol. Co., Ltd., Beijing, China \\ Email: hello_liujia@126.com, changjunren@huawei.com
}

Received June, 2013

\begin{abstract}
With the growing concern on data rates and resource utilization, Device-to-Device (D2D) communication has been raised in 3GPP Long-Term Evolution (LTE) networks. In order to limit severe interference, previous studies mainly focus on intra-cell interference that between cellular links and local D2D links. In this paper, we consider both intra-cell interference and inter-cell interference between D2D and cellular links. We propose a new resource reuse algorithm that D2D users reuse the minimum interference uplink (UL) Semi-Persistent Scheduling (SPS) resources to reach the highest throughput. The simulation results show that this scheme reduces interference as well as improves throughput.
\end{abstract}

Keywords: Device-to-device; Inter-cell Interference; Interference Reduction; Semi-Persistent Scheduling; Resource Sharing

\section{Introduction}

Device-to-device (D2D) communication as an underlay coexisting with cellular networks has been proposed in order to improve the utilization of the spectrum [1-4]. There are four resource allocation methods in D2D communication underlaying cellular networks: cellular mode, dedicated resource mode, reusing the resource of only one cellular user, and reusing the resources of more than one cellular user [4]. The first two modes bring no interference to system because they use orthogonal resource. In the last two modes which belong to reuse mode, D2D users share the same resource with cellular users, so they interfere each other. The interference includes intra-cell interference (the interference between attached cellular users and D2D users) and inter-cell interference (the interference between neighbor cellular users and attached cellular users; the interference between neighbor cellular users and attached D2D users; the interference between neighbor D2D users and attached cellular users; the interference between neighbor D2D users and attached D2D users). However, the network may achieve maximum resource utilization in reuse mode.

When D2D users share the downlink (DL) resource with cellular user, cellular users and neighbor cellular users may suffer from interference by D2D users, and

\footnotetext{
*This work was supported by the Huawei Innovation Research Program, the Fundamental Research Funds for the Central Universities (K5051301034), and the 111 Project (B08038).
}

D2D receiver may suffer from interference by eNodeB (eNB). On the other hand, when D2D users share the uplink (UL) resource with cellular, the eNodeB is the victim of interference by D2D users, and D2D receiver may suffer from interference by cellular users and neighbor cellular users.

In order to reduce these interferences, a lot of work have been done [5-11]. In [5], Xiao xiao proposes a power optimization scheme with joint resource allocation and mode selection in an OFDM system with integrated D2D communications, aiming at optimizing downlink power consumption. In [6], Yu considers rate splitting and interference cancelation in D2D communication underlaying a cellular network. They assume that a transmitted message is split into a private and a public part, using fractions $\alpha_{i}$ and $1-\alpha_{i}$ of the total transmit power, respectively. They also derive the optimal rate splitting factors for most of the categorized channel conditions in a two-link scenario. In [7], Janis, P. Koivunen proposes a practical and efficient scheme where the D2D terminals make power measurements during the uplink (UL) phase of the cellular network in normal operation. In [8], $\mathrm{H}$. Wang and $\mathrm{X}$. Chu propose a distance-constrained resource-sharing criterion for the base station to select a cellular user for a D2D link, with the cellular-to-D2D interference controlled by keeping a minimum distance between them. In [9], the joint mode selection and power allocation scheme is proposed to maximize the utility function. In [10], Zulhasnine, M. formulates the problem 
of RB allocation to the D2D communication as a mixed integer nonlinear programming (MINLP), and proposes an alternative greedy heuristic algorithm to solve it. In [11], Interference Alignment (IA) is used in a D2D underlay network to enhance spectral efficiency. But in these papers, authors do not consider inter-cell interference to D2D users.

In this paper, we consider both intra-cell interference and inter-cell interference between D2D and cellular links in D2D reuse mode. A new resource reuse algorithm that D2D user reuse the UL SPS resource is proposed. We choose the minimum interference resource to reach high throughput. It's shown that our algorithm can bring less interference and higher throughput.

The rest of this paper is organized as follows. In Section 2 we give the system model of D2D communication underlay LTE network. In Section 3, we formulate our resource algorithm and mechanism. In Section 4, some numerical results are given. Finally, some concluding remarks are drawn in Section 5.

\section{System Model}

In this paper, we assume D2D users reuse LTE UL SPS (Semi-Persistent Scheduling) resource. When D2D users reuse UL resource, D2D receiver may suffer from interference by attached cellular users, neighbor cellular users, and neighbor D2D users. An example of interference scenario in the uplink is given in Figure 1. CUE denotes cellular users and DUE denotes D2D users. D2D users are in anchored eNB (A-eNB) and its neighbor eNB is NeNB. Communication links are indicated by the solid line while interference links are indicated by the dotted line. DUE2 may suffer from interference by $\mathrm{CUE}_{1}^{1}, \mathrm{CUE}_{1}^{2}$, $\mathrm{CUE}_{1}^{3}, \mathrm{CUE}_{2}^{1}, \mathrm{CUE}_{2}^{2}, \mathrm{CUE}_{2}^{3}$ and DUE3. And when $\mathrm{CUE}_{1}^{1}$ transmit to A-eNB, A-eNB may suffer from interference by DUE1, $\mathrm{CUE}_{2}^{1}$ and DUE3. As in Figure 1 the interference of $\mathrm{CUE}_{2}^{1}$ in $\mathrm{N}$-eNB to D2D link is strong, so we should avoid choosing the same resource of $\mathrm{CUE}_{2}^{1}$ for D2D link.
Some useful symbols are defined as:

\begin{tabular}{ll}
\hline$P_{d}, P_{c}$ & $\begin{array}{l}\text { The transmission power of D2D user and cel- } \\
\text { lular user }\end{array}$ \\
$G_{d d}$ & A channel gain of D2D link \\
$G_{c B}$ & A channel gain of cellular link \\
$y_{i}^{j}$ & $\quad \begin{array}{l}\text { A binary variable which satisfies } y_{i}^{j}=1 \text { if user } \\
N_{o}\end{array}$ \\
$i$ & Noise power at the receiver $j$ \\
$j$ & User device index in attached cell \\
\end{tabular}

Considering intra-cell interference and inter-cell interference, the UL SINR of D2D link and cellular link can be expressed by:

$$
\begin{aligned}
& \gamma_{d}^{U L}=\frac{P_{d} G_{d d}}{N_{0}+\sum_{i} y_{i}^{d} P_{i} G_{i d}+\sum_{j} y_{j}^{d} P_{j} G_{j d}+\sum_{d} y_{d}^{D} P_{d} G_{d d}} \\
& \gamma_{e N B}^{c}=\frac{P_{c} G_{c B}}{N_{0}+\sum_{i} y_{i}^{c} P_{i} G_{i B}+\sum_{d} y_{i}^{d} P_{d} G_{d d}+\sum_{j} y_{j}^{c} P_{j} G_{j B}}
\end{aligned}
$$

Because SPS resource has its own regularity, we choose it to reuse. SPS is a feature that significantly reduces control channel overhead for applications that require persistent radio resource allocations such as VoIP. It means that the size of packets and the arriving time intervals are constant over a period of time, and users are allocated with resource periodically. The period length is the time that a traffic occupies resource periodically. Usually, the algorithm of resource selection might take some time. The most likely scenario is that we compute RB (i) of user A is reasonable to reuse, but cannot reuse $\mathrm{RB}$ (i) because this RB is not assigned to user $A$ when starting the step of sharing resource. Using SPS resource can solve this problem due to its fixed resource assignment in a period of time.

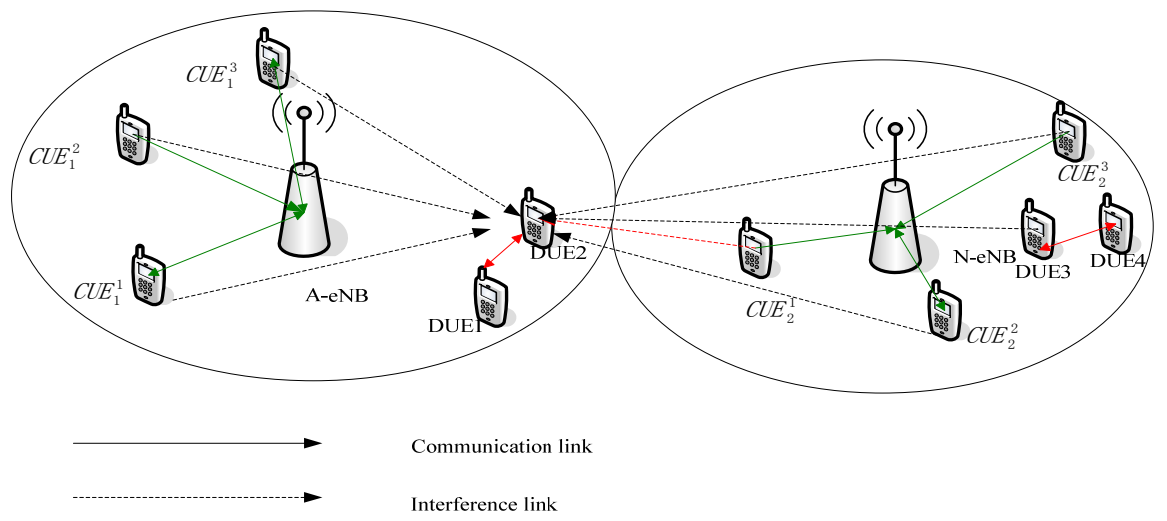

Figure 1. An example of interferences in the LTE uplink. 
Taking typically VoIP as an example, the packets arriving time interval is $20 \mathrm{~ms}$. eNB gives SPS scheduling indication to users through PDCCH, then users can transmit or receive data in this schedule and transmit or receive new VoIP data on the same resource after every 20 ms. The SPS resource schedule is given in Figure 2.

In this paper, we assume eNBs can exchange SPS resource allocation information through X2 interface. A-eNB controls D2D pairs reuse SPS resource, consults with N-eNBs, and measures correlates, then selects the most appropriate SPS resource to reuse.

\section{Resource Reuse Algorithm Considering Inter-cell Interference}

In this section, we will describe the SPS resource reuse algorithm in details. DUE1 and DUE2 define D2D users. The proposed scheme is presented as follows.

Step 1: All cellular users and D2D users register to its anchored eNB (A-eNB) and A-eNB reports its SPS resource using information to its neighbor eNBs (N-eNBs) by interface $\mathrm{X} 2$.

Step 2: According to the information obtained from interface X2, N-eNBs decide which resource can be used as SPS resource. In this way, A-eNB and N-eNBs would use the same SPS resource.

Step 3: D2D users (DUE1 and DUE2) report their position information to A-eNB.

The position information can be obtained from GPS (Global Position System) or A-GPS (Assisted GPS). For example, the coordinates of DUE1 and DUE2 are $\left(x_{1}, y_{1}\right)$, $\left(x_{2}, y_{2}\right)$. If the distance between DUE1 and DUE2 $d_{1,2}<d_{0} \quad\left(d_{0}\right.$ is the threshold), and the channel condition is good (we can use a detecting signal, and judge if the block error rate (BLER) is less than a threshold), they can form a D2D pair, and then go to next Step. As what is said above,

$$
d_{1,2}=\sqrt{\left(x_{2}-x_{1}\right)^{2}+\left(y_{2}-y_{1}\right)^{2}}
$$

Step 4: A-eNB computes the distance between cellular users and D2D users, choose $n$ (e.g. three) maximum distance, and takes their corresponding SPS resource as candidates. The distance between cellular users and D2D pairs is

$$
d_{c, d}=\sqrt{\left(x_{c}-\frac{x_{1}+x_{2}}{2}\right)^{2}+\left(y_{c}-\frac{y_{1}+y_{2}}{2}\right)^{2}}
$$

Step 5: A-eNB reports these n SPS resource groups and D2D positions to N-eNB.

Step 6: N-eNB searches its cell for users who use the same resource, and then orders the corresponding users compute the interference to neighbor D2D users on the SPS resource.

Step 7: N-eNB receives the interference information from the chosen users in step6 and gives feedback to A-eNB.

Step 8: A-eNB searches the smallest interference and choose its corresponding resource as the SPS resource to reuse.

Step 9: A-eNB allocates the SPS resource group to D2D users.

Step 10: D2D signaling and data can be transmitted between DUE1 and DUE2.

The complete procedure of our proposed algorithm can be illustrated in Figure 3.

It should be pointed out that, A-eNB may have several neighbors, and we should consider several N-eNBs reality.

Taking Figure 1 as an example. If the distance between two users $d_{i, j}<d_{0}$ ( $d_{0}$ is a distance threshold), they can form a D2D pair (DUE1, DUE2). CUE 1 , $\mathrm{CUE}_{1}^{2}, \mathrm{CUE}_{1}^{3}$ are three cellular users in A-eNB, which are farthest to the D2D pairs. The RB groups (RBG) assigned to them are RBG (1), RBG (2), and RBG (3), respectively. In the neighbor cell, $\left(\mathrm{CUE}_{2}^{1}, \mathrm{CUE}_{2}^{2}, \mathrm{CUE}_{2}^{3}\right)$ use this three RB groups. A-eNB and N-eNB exchange their SPS resource allocation information through X2 interface. Assuming the transmitting power of user $i$ is $P_{i}$, and the pathloss between cellular user $i$ and D2D user is $P L_{i}^{d}$. Then compute $\min \left\{P_{2 i}^{t} / P L_{2 i}^{d_{2}}, i=1,2,3\right\}$ and find $i$. So RBG (i) can be assigned to D2D pairs. Then D2D users can communication on this RBG.

\section{Simulation and Performance Analysis}

In this section, simulation results are shown to evaluate the performance of a hybrid system by using our idea.

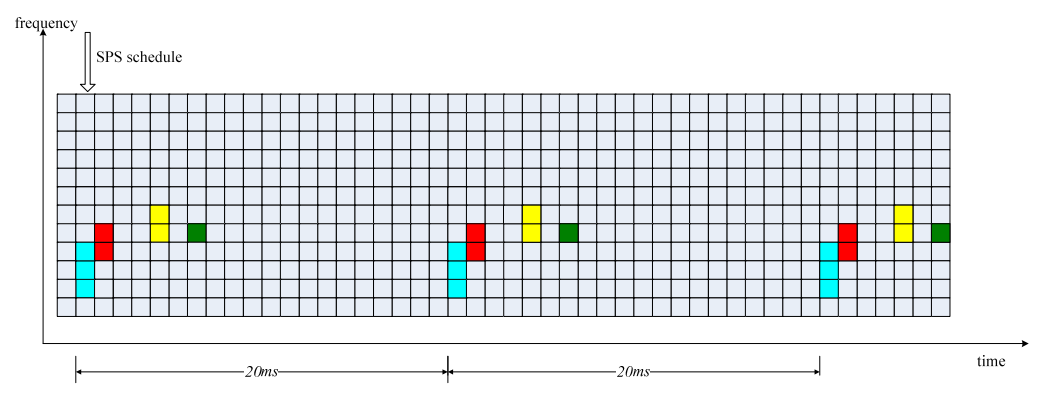

Figure 2. The SPS resource schedule. 


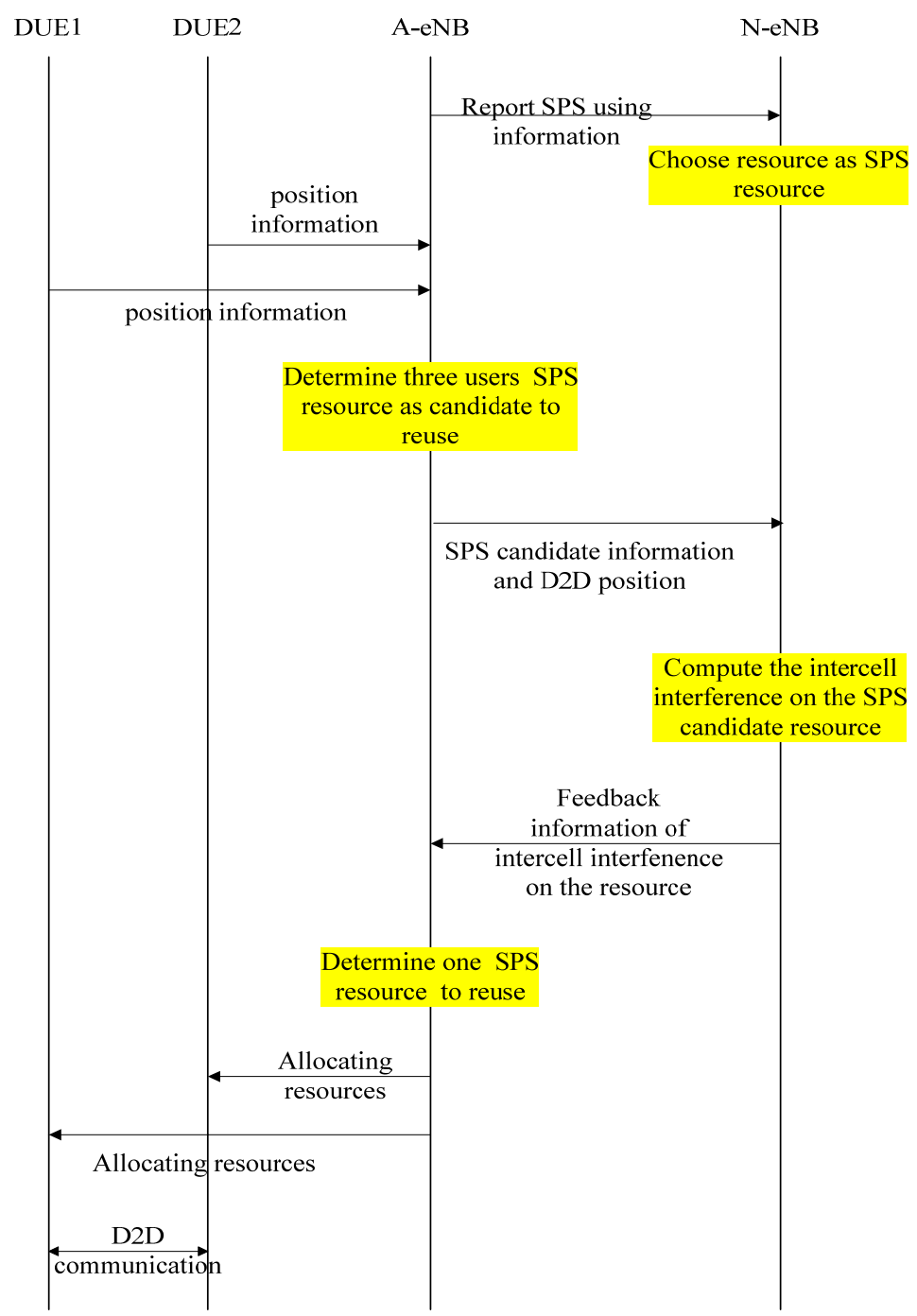

Figure 3. The proposed SPS resource reuse scheme.

\subsection{Simulation Parameters}

We consider a 7 hexagonal wrap-around-cell layout. Cellular users are randomly located in cells and D2D users are located in cells' edge. When computing the inter-cell interference to D2D link, the 6 cells around should be considered. The distance between D2D users is less than $50 \mathrm{~m}$ and the distance between two eNBs is $500 \mathrm{~m}$. There are 30 cellular users and 6 D2D pairs in each cell. In this simulation, the 3-sector antenna is used for each eNB. LTE power control scheme [13] is utilized by controling the power of cellular users and a constant is used to express the power of D2D users. The setting of the UE transmit power for the physical uplink shared channel (PUSCH) transmission in subframe i is defined by

$$
\begin{aligned}
& P_{\mathrm{PUSCH}}(i)=\min \left\{P_{\mathrm{CMAX}}, 10 \log _{10}\left(M_{\mathrm{PUSCH}}(i)\right)\right. \\
& \left.+P_{\mathrm{O} \_ \text {PUSCH }}(j)+\alpha(j) \cdot P L+\Delta_{\mathrm{TF}}(i)+f(i)\right\}
\end{aligned}
$$

where,

- $P_{\text {CMAX }}$ is the configured maximum UE transmitted power.

- $M_{\text {PUSCH }}(i)$ is the bandwidth of the PUSCH resource assignment expressed in number of resource blocks valid for subframe i.

- $P_{O \quad P U S C H}(j)$ is a parameter composed of the sum of a cell specific nominal component.

- For $\mathrm{j}=0$ or $1, \alpha \in\{0,0.4,0.5,0.6,0.7,0.8,0.9,1\}$ is a 3-bit cell specific parameter provided by higher layers.

- PL is the downlink pathloss estimate calculated in the UE in $\mathrm{dB}$.

- $\Delta_{T F}(i)=10 \log \left(\left(2^{\text {MPR.K } K_{S}}-1\right) \beta_{\text {offset }}^{\text {PUSCH}}\right)$ for $K_{S}=1.25$ and 0 for $K_{S}=0$ where $K_{S}$ is given by the UE specific parameter deltaMCS-Enabled provided by higher layers.

- $\delta_{\text {PUSCH }}$ is a UE specific correction value, $f(i)=$ $f(i-1)+\delta_{\text {PUSCH }}\left(i-K_{\text {PUSCH }}\right)$. 
There are three kind of path loss in a D2D scenario [12]. The path loss model between cellular users and eNB is COST 231 Hata model, giving

$$
\text { pathloss }(d B)=36.7+35 \times \lg (d)
$$

The path loss between cellular users and D2D users is Xia model, giving

$$
\text { pathloss }=\left\{\begin{array}{c}
66.5+40 \times \lg (d), d>50 \\
100.7+20 \times \lg (d), d<50
\end{array}\right.
$$

And the path loss between two D2D users is free space model, giving

$$
\text { pathloss }=38.4+20 \times \lg (d)
$$

$d$ is link distance in meter. The left parameters are presented in Table 1.

\subsection{Simulation Results and Discussions}

We will show some simulation results to confirm our method's advantages in this part.

Figure 4 compares the sum throughput of system only with cellular users, the system with cellular users and D2D users that not handle inter-cell interference, and the system with cellular users and D2D users using the

\begin{tabular}{|c|c|c|}
\hline \multicolumn{2}{|c|}{ Parameter } & Value \\
\hline \multicolumn{2}{|c|}{ Noise Power Density } & $-174 \mathrm{dBm} / \mathrm{Hz}$ \\
\hline \multicolumn{2}{|c|}{ RB bandwidth } & $180 \mathrm{kHz}$ \\
\hline \multicolumn{2}{|c|}{ Carrier frequency } & $2 \mathrm{GHz}$ \\
\hline \multicolumn{2}{|l|}{ RB number } & 100 \\
\hline \multicolumn{2}{|c|}{ Max UE Power } & $200 \mathrm{~mW}$ \\
\hline \multicolumn{2}{|c|}{ Min UE Power } & $3.2 \mathrm{~mW}$ \\
\hline \multicolumn{2}{|c|}{ D2D UE Power } & $2 \mathrm{~mW}$ \\
\hline \multicolumn{2}{|c|}{ Max BS antenna gain } & $14 \mathrm{dbi}$ \\
\hline \multicolumn{2}{|c|}{ UE antenna gain } & 0 \\
\hline \multicolumn{2}{|c|}{ Shadowing standard deviation } & $8 \mathrm{~dB}$ \\
\hline \multirow{2}{*}{$\begin{array}{l}\text { Shadowing } \\
\text { correlation }\end{array}$} & Between cells & 0.5 \\
\hline & Inner cell & 1 \\
\hline \multirow{2}{*}{\multicolumn{2}{|c|}{$\begin{array}{l}\text { Antenna pattern (horizontal) } \\
\text { (For 3-sector cell sites with } \\
\text { fixed antenna patterns) }\end{array}$}} & $A(\theta)=-\min \left[12\left(\frac{\theta}{\theta_{3 d B}}\right)^{2}, A_{m}\right]$ where \\
\hline & & $\theta_{3 d B}=70$ degrees, $A_{m}=20 \mathrm{~dB}$ \\
\hline \multicolumn{2}{|c|}{ BS noise figure } & $5 \mathrm{~dB}$ \\
\hline \multicolumn{2}{|c|}{$\begin{array}{l}\text { Modulation and coding } \\
\text { scheme (MCS) }\end{array}$} & QPSK, 16QAM,64QAM \\
\hline \multicolumn{2}{|c|}{ Number of symbols per slot } & 7 (1 Pilot +6 Data) \\
\hline
\end{tabular}
proposed method. We observe that the system throughput

Table 1. System parameters. can be significantly improved by using D2D communication. When using our resourse reuse algorithm considering neighbor cell interference, the throughput is higher than traditional algorithm.

The focus of our study is that the interference to D2D users can be reduced and the throughput of cellular do not be significantly reduced. This can be validated in Figure 5 and Figure 6. Because D2D users are distributed in each cell edge and they share the same reuse with cellular users which are far from them, the interference to from D2D users to cellular users is small. Figure 7 shows the throughput per cellular user in the three cases above. It can be seen that no matter handle neighbor cell interference or not, cellular users' throughput changes little. Figure 8 shows the interference received by cellular users in the three cases above. The cellular users' interference stays the same. So we can conclude that our algorithm has little effect on cellular users.

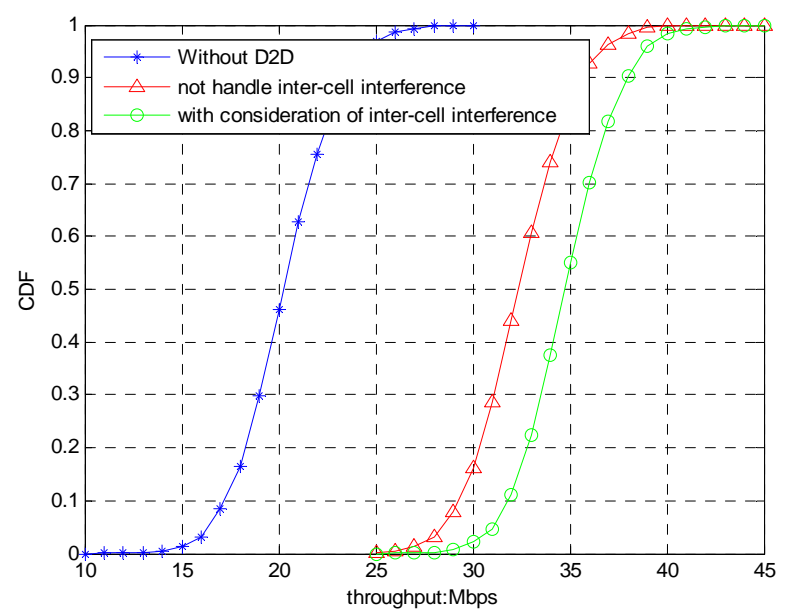

Figure 4. The sum throughput of system only with cellular users, the system with cellular users and D2D users that not handle inter-cell interference, and the system with cellular users and D2D users using the proposed method.

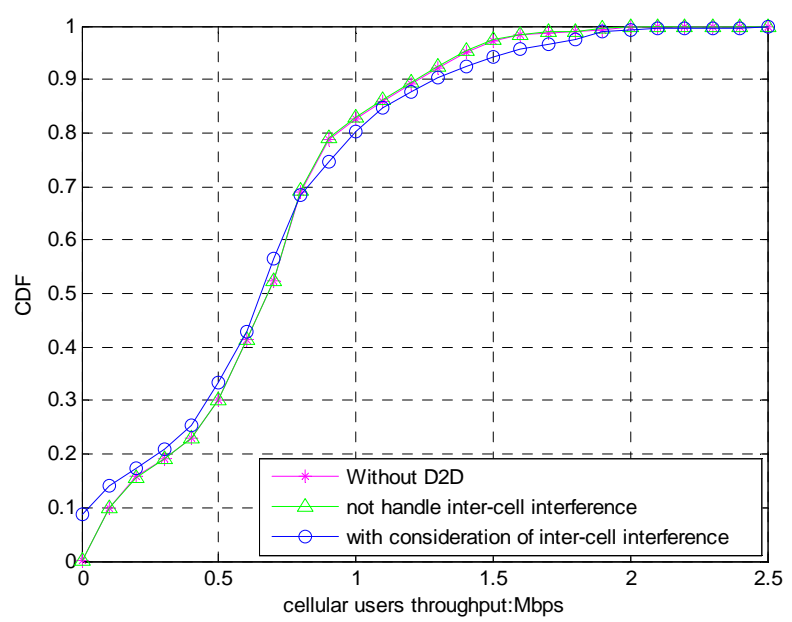

Figure 5. The throughput per cellular user. 


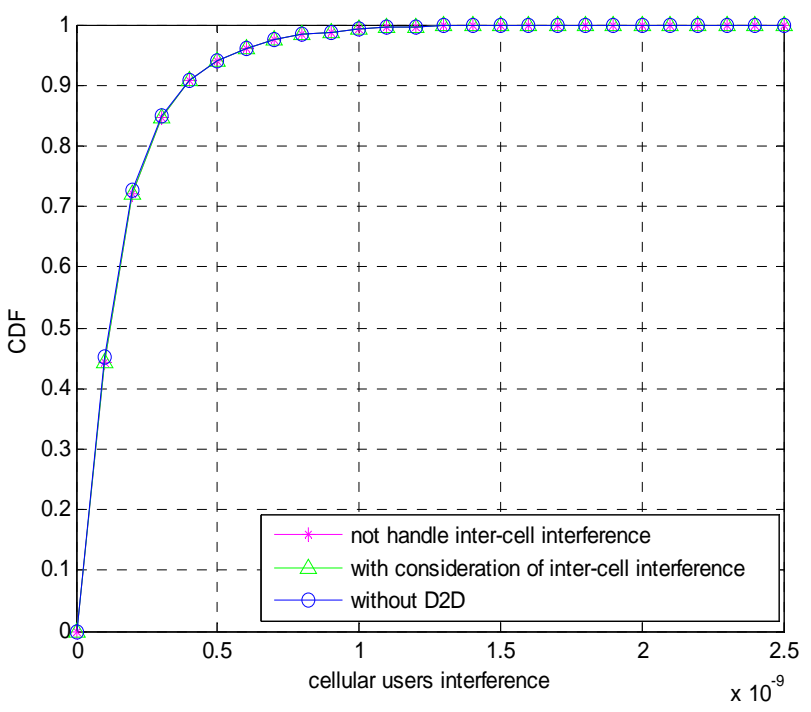

Figure 6. The interference of cellular users.

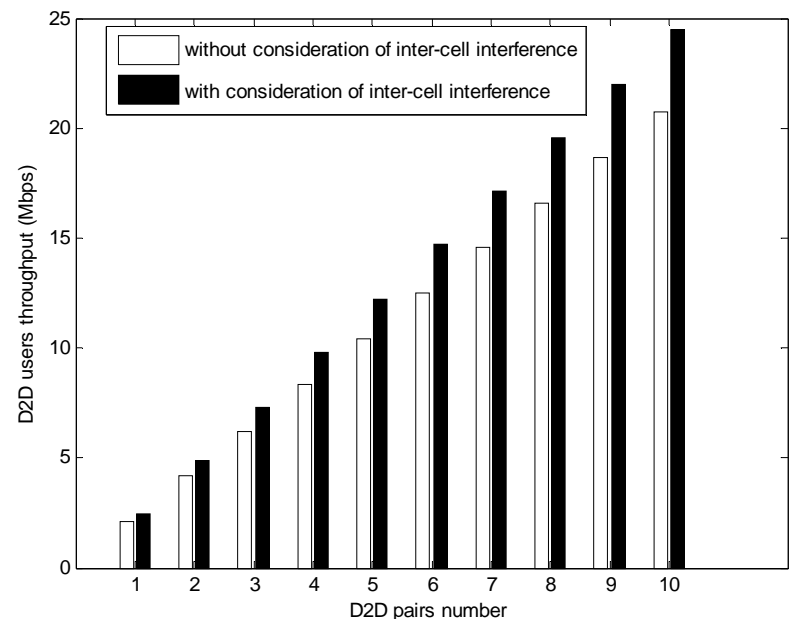

Figure 7. The D2D throughput with D2D number changed.

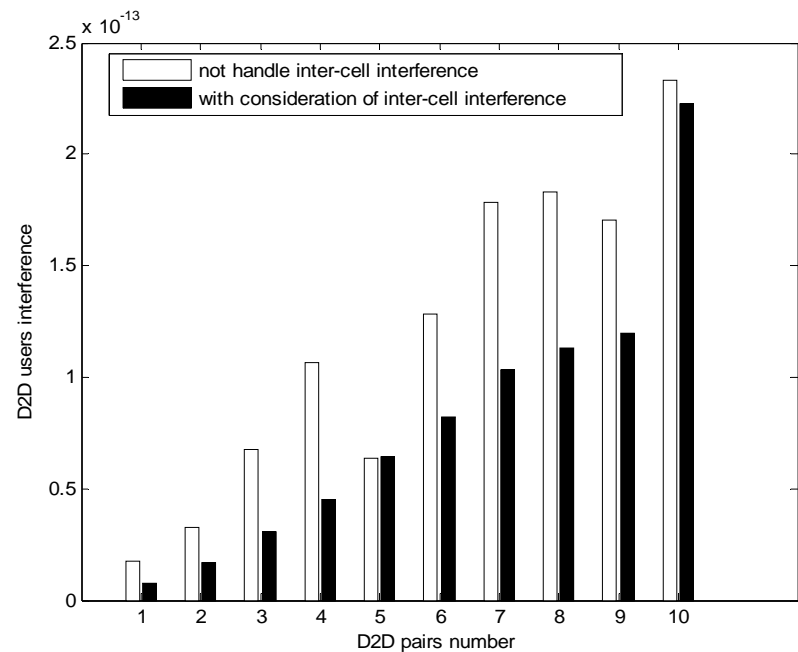

Figure 8. The interference of D2D users with D2D number changed.
To investigate the interference D2D users received, we change D2D pairs' number from 1 to 10 , and plot the D2D users' interference (Figure 8). When D2D pairs' number is small, the interference to D2D users is small, too. But when the number increases, D2D users may suffer from high interference by cellular users and neighbor cellular users. It's shown that our algorithm greatly reduced the interference to D2D users.

\section{Conclusions}

In this paper, we proposed a new resource reuse algorithm that D2D user reuse the UL SPS resource. We consider both intra-cell interference and inter-cell interference between D2D and cellular links, and it's more closely to practical conditions. By choosing the reasonable resource to reuse, the systerm throughput grows higher. Simulation results show that the proposed algorithm significantly improves systerm throughput but causes little effect to the cellular users' throughput. When we use the proposed algorithm, the D2D throughput can be increased and the interference to D2D links is reduced.

\section{REFERENCES}

[1] K. Doppler, M. P. Rinne, P. Janis, C. Ribeiro and K. Hugl, "Device-to-Device Communications; Functional Prospects for LTE-Advanced Networks,” ICC Workshops, 14-18 June 2009.

[2] S. Hakola, T. Chen, J. Lehtomaki and T. Koskela, “Device-to-Device (D2D) Communication in Cellular Network-Performance Analysis of Optimum and Practical Communication Mode Selection," Wireless Communications and Networking Conference (WCNC), 18-21 April 2010.

[3] G. Fodor and N. Reider, “A Distributed Power Control Scheme for Cellular Network Assisted D2D Communications,” Global Telecommunications Conference (GLOBECOM 2011), 5-9 Dec. 2011.

[4] B. Wang, L. Chen, X. H. Chen, X. Zhang and D. C. Yang, "Resource Allocation Optimization for Device-to-Device Communication Underlaying Cellular Networks," Vehicular Technology Conference (VTC Spring), 15-18 May 2011.

[5] X. Xiao, X. M. Tao and J. H. Lu, "A QoS-Aware Power Optimization Scheme in OFDMA Systems with Integrated Device-to-Device (D2D) Communications," Vehicular Technology Conference (VTC Fall), 5-8 Sept. 2011.

[6] C.-H. Yu and O. Tirkkonen, "Device-to-Device Underlay Cellular Network Based on Rate Splitting," Wireless Communications and Networking Conference (WCNC), 1-4 April 2012.

[7] P. Janis, V. Koivunen, Ribeiro, etc. “Interference-aware Resource Allocation for Device-to-device Radio underlaying Cellular Networks," Vehicular Technology Conference, 26-29 April 2009. 
[8] H. Wang and X. Chu, "Distance-constrained Resource-sharing Criteria for Device-to-device Communications Underlaying Cellular Networks," Electronics Letters , Vol. 48, No. 9, 2012, pp. 528-530.

[9] M. Jung, K. Hwang and S. Choi, "Joint Mode Selection and Power Allocation Scheme for Power-Efficient Device-to-Device (D2D) Communication," Vehicular Technology Conference (VTC Spring), 6-9 May, 2012.

[10] M. Zulhasnine, C. C. Huang and A. Srinivasan, "Efficient Resource Allocation for Device-to-Device Communication Underlaying LTE Network," Wireless and Mobile Computing, Networking and Communications (WiMob), 11-13 Oct. 2010, pp. 368-375.
[11] Elkotby, H. E. Elsayed, Ismail and M.H., "Exploiting Interference Alignment for Sum Rate Enhancement in D2D-Enabled Cellular Networks," Wireless Communications and Networking Conference (WCNC), 1-4 April 2012, pp. 1624 -1629.

[12] X. Y. Zhu, S. Wen, etc., “A Cross-layer Study: Information Correlation Based Scheduling Scheme for Device-to-Device Radio Underlaying Cellular Networks," International Conference on Telecommunications (ICT), 23-25 April 2012.

[13] "Evolved Universal Terrestrial Radio Access (E-UTRA); Physical Layer Procedures (Release 9),” 3GPP TS 36.213 V9.2.0, 2010. 